\title{
A mean-field modeling study of the interaction between hydrogen and a palladium (110) single crystal
}

\author{
M. Mavrikakis, J. W. Schwank, and J. L. Gland \\ Department of Chemical Engineering, The University of Michigan, Ann Arbor, Michigan 48109-2136
}

(Received 14 June 1994; accepted 6 August 1996)

\begin{abstract}
A new interpretation of the temperature programmed desorption (TPD) spectra of hydrogen on a $\operatorname{Pd}(110)$ single crystal surface is presented. The transient mean-field model developed accounts for both adsorption on the surface and diffusion of hydrogen into the lattice of the crystal. A new approach for modeling TPD spectra is developed, in which both the isothermal exposure as well as the temperature ramp of the TPD experiment are modeled in a consistent way. We demonstrate that for systems with substantial diffusion of the adsorbate into the substrate's lattice it is not sufficient to report exposure values. For such systems, exposure time has a far more pronounced effect than the exposure pressure has on the TPD spectra, because diffusion is a strongly time-dependent process. The modeling and experimental results for the $\mathrm{H}_{2} / \mathrm{Pd}(110)$ system are in good agreement and suggest the existence of a distinct subsurface state, in addition to the surface and bulk states of hydrogen. Low frequency factors derived from the model for the processes connecting the subsurface with the surface and bulk state emphasize the restricted nature of the corresponding transition states. (C) 1996 American Institute of Physics. [S0021-9606(96)02242-8]
\end{abstract}

\section{INTRODUCTION}

Systems coupling kinetics (reaction) and transport (diffusion) processes are classified as RD systems. Kinetic expressions are time-dependent only, whereas diffusion processes must be described in terms of both time and space. Thus, RD systems exhibit both temporal as well as spatial evolution. Typical examples include the model proposed for the explanation of the Belousov-Zhabotinsky (BZ) reaction patterns ${ }^{1-4}$ and the model suggested for the rationalization of the patterns on leopard's skin. ${ }^{5,6}$ Recently, substantial progress has been made in understanding RD systems by applying nonlinear theory ${ }^{1,2,7-11}$ and making use of advances in both computational speed and methods. ${ }^{12-14}$ The main objective of this contribution is to propose a simple RD model to account for the coupling between surface kinetic processes and diffusion of hydrogen in the bulk of a $\mathrm{Pd}(110)$ single crystal. Our goal is to model the TPD spectra of hydrogen and compare the predicted spectra with the experimental results.

TPD methods have been extensively used for studying the surface processes dominating gas-solid interactions over a significant pressure and temperature range. TPD spectra are characteristic of the gas-solid system being studied and give valuable information about the processes involved. Excellent reviews ${ }^{15,16}$ give the details of a typical TPD experimental protocol and describe common methods used for analyzing the data. Traditional methods for extracting mechanistic information from TPD spectra are restricted by several simplifying assumptions. Previous models either assumed that the important reservoirs (surface and bulk) were well-mixed or used semi-infinite medium formulations of the mathematical problem. ${ }^{17}$ Each of these two approaches offers substantial insight regarding either the TPD spectra or the adspecies uptake mechanism, but neither of these approaches attempts to connect the two problems. Only recently have we pro- posed a rigorous mathematical approach ${ }^{18-20}$ for the analysis of TPD spectra of systems with strong coupling between kinetic processes on the surface and diffusive processes in the lattice of the solid. The major contribution of that work is the elimination of the assumption of well-mixed reservoirs, the finite medium formulation of the problem, and the consistent quantitative treatment of all the individual experimental phases. The model introduced in the present study extends and applies a coupled RD approach to the $\mathrm{H}_{2} / \mathrm{Pd}(110)$ system.

In the sections to follow, after we describe this new model, calculated desorption spectra are compared with the available experimental data. We conclude by proposing a one-dimensional potential energy diagram as well as values for the physical parameters of our model that give calculated TPD spectra in good agreement with the experimental results. Good agreement between the calculated and the experimental TPD spectra was achieved only when a distinct subsurface state was incorporated in our model. As a result, the model outlined in the next section incorporates three distinct hydrogen states: surface, subsurface, and bulk.

\section{MATHEMATICAL MODEL AND SOLUTION METHODOLOGY}

Our general RD models ${ }^{18,19}$ considered surface and bulk as the only distinct domains in a single crystal. For the case of $\mathrm{H}_{2} / \mathrm{Pd}(110)$, such a simple two domain model proved to be inadequate ${ }^{20}$ for the accurate description of the experimental results. Despite our best efforts, a model incorporating only surface and bulk states could not yield TPD spectra in reasonable agreement with the available experimental data. Furthermore, previous workers ${ }^{21}$ had speculated from low energy electron diffraction (LEED) experiments that the concentration of dissolved hydrogen atoms is enhanced within the layers just below the surface. Others have also 


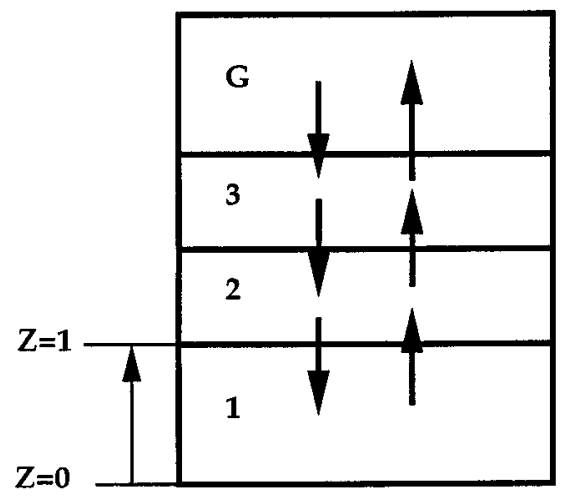

FIG. 1. Division of the Pd(110) single crystal into surface (3), subsurface (2), and bulk (1) layers. Arrows indicate rate processes connecting the three layers, and coupling surface with the gas phase $(\mathrm{G})$.

supported the idea of a separate subsurface state for hydrogen in palladium, based on theoretical calculations totally independent of any TPD behavior. ${ }^{22}$ Thus, we propose and test here the existence of a third, distinct domain, namely the subsurface of the single crystal (Fig. 1). Surface is the first atomic layer of the solid, while subsurface consists of the few atomic layers immediately beneath the surface. The rest of the crystal, consisting of thousands of atomic layers, is defined as the bulk of the solid. This is the first time that the existence of a distinct subsurface state coupled with both bulk and surface states, has been evaluated within the framework of the TPD behavior of the $\mathrm{H}_{2} / \mathrm{Pd}$ system.

Surface and subsurface are treated as spatially uniform domains of zero thickness. Fickian diffusion governs the migration of species into the bulk leading to one-dimensional concentration gradients along the thickness dimension of the crystal. No concentration gradients are allowed across each horizontal partition of the bulk. Further assumptions of this model include: (i) the gas-phase pressure remains constant during the exposure; (ii) the temperature of the entire crystal is uniform at any given time; (iii) physical parameters of the system appearing in the model, such as activation energies, pre-exponential factors, and all three site densities are constant, independent of temperature and hydrogen concentration; (iv) adsorption on the surface is nonactivated and dissociative, while desorption is activated and recombinative; (v) surface structure remains unchanged, since all temperatures used here are well above the $\operatorname{Pd}(110)$ surface reconstruction temperature $(\sim 200 \mathrm{~K}){ }^{23}$

Based on these assumptions, the model proposed for the interpretation of the TPD spectra of the $\mathrm{H}_{2} / \mathrm{Pd}(110)$ system is formulated as follows. Equations (1) and (2) represent the transient mass balance for the surface and subsurface layers, respectively,

$$
\begin{aligned}
& \frac{d X_{3}}{d \tau}=\left(\frac{L^{2}}{D_{0}}\right)\left\{R_{23}-R_{32}+R_{a}-R_{d}\right\}, \\
& \frac{d X_{2}}{d \tau}=\left(\frac{L^{2}}{D_{0}}\right)\left\{R_{12}-R_{21}+\left[\frac{X_{3 s}}{X_{2 s}}\right]\left(R_{32}-R_{23}\right)\right\} .
\end{aligned}
$$

$X_{3}$ and $X_{2}$ represent the normalized surface and subsurface concentrations of hydrogen, with $X_{3 s}$ and $X_{2 s}$ denoting the corresponding normalization constants, i.e., the surface and subsurface site density, respectively. The processes of adsorption $\left(R_{a}\right)$, desorption $\left(R_{d}\right)$, migration from surface to subsurface and vice versa $\left(R_{32}\right.$ and $\left.R_{23}\right)$, directly determine surface concentration. Similarly, the processes determining subsurface concentration are those connecting subsurface to surface $\left(R_{23}\right.$ and $\left.R_{32}\right)$ and subsurface to bulk $\left(R_{12}\right.$ and $\left.R_{21}\right)$. Each one of the rate processes connecting surface, subsurface, and bulk is assumed to be activated as shown in the following expressions:

$$
\begin{aligned}
& R_{23}=F_{23} \exp \left(\frac{-E_{23}^{*}}{R T(\tau)}\right) X_{2}\left(1-X_{3}\right), \\
& R_{21}=F_{21} \exp \left(\frac{-E_{21}^{*}}{R T(\tau)}\right) X_{2}\left(1-Y_{Z=1}\right), \\
& R_{12}=F_{12} \exp \left(\frac{-E_{12}^{*}}{R T(\tau)}\right) Y_{Z=1}\left(1-X_{2}\right), \\
& R_{32}=F_{32} \exp \left(\frac{-E_{32}^{*}}{R T(\tau)}\right) X_{3}\left(1-X_{2}\right) .
\end{aligned}
$$

$F_{i j}$ and $E_{i j}^{*}$ represent frequency factors and activation energies of the corresponding rate processes. $Y_{Z=1}$ denotes the normalized hydrogen concentration at the topmost layer of the bulk $(Z=1)$. The corresponding normalization constant is the site density of the bulk $\left(Y_{s}\right)$. Expressions (7) and (8) give the adsorption and desorption rates, respectively,

$$
\begin{aligned}
& R_{a}=S_{0} P\left(1-X_{3}\right)^{2}\left(\frac{2}{X_{3 s}}\right)\left[\frac{1}{2 \pi m k T(\tau)}\right]^{1 / 2}, \\
& R_{d}=F_{d} \exp \left(\frac{-E_{d}^{*}}{R T(\tau)}\right) X_{3}^{2} X_{3 s},
\end{aligned}
$$

where $S_{0}$ is the initial sticking coefficient, $P$ is the gas-phase pressure, $k$ is Boltzmann's constant, $m$ is the atomic mass of hydrogen, $F_{d}$ and $E_{d}^{*}$ are the desorption frequency factor and activation energy, respectively, and $T(\tau)$ is the temperature of the crystal as a function of dimensionless time $(\tau)$, which is defined as $\left(D_{0} / L^{2}\right) * t$, where $t$ is real time. $L$ and $Z$ denote the real and dimensionless thickness of the bulk, correspondingly. $D_{0}$ and $E_{D}^{*}$ are the pre-exponential factor and activation energy of the Fickian diffusion process in the bulk.

The evolution of hydrogen concentration $(Y)$ into the bulk of the crystal, as a function of both time and space, is described by Eq. (9)

$$
\frac{\partial Y}{\partial \tau}=\exp \left(\frac{-E_{D}^{*}}{R T(\tau)}\right) \frac{\partial^{2} Y}{\partial Z^{2}},
$$

which is valid for all $\tau>0$ and $0<Z<1$, whereas the boundary conditions for its solution are: zero flux at the bottom of the bulk $(Z=0)$, and 


$$
\left(\frac{\partial Y}{\partial Z}\right)_{Z=1}=\frac{L}{D_{0} \exp \left(-E_{D}^{*} / R T(\tau)\right)}\left(\frac{X_{2 s}}{Y_{s}}\right)\left[R_{21}-R_{12}\right]
$$

representing flux continuity at the subsurface-bulk interface.

A linear temperature ramp, with a constant heating rate $\beta$, is formulated as follows:

$$
\frac{d T}{d \tau}=\left(\frac{L^{2}}{D_{0}}\right) \beta
$$

Equations (1), (2), (9), and (11) are simultaneously integrated for each individual step in the adsorption-desorption experiment. The simulation of the isothermal exposure begins with an identically zero concentration profile for the bulk, subsurface, and surface layers. The adsorption temperature, exposure pressure, and time are specified according to the experimental protocol used. The concentration profile calculated at the end of the isothermal exposure simulation is used as the initial concentration profile for the simulation of the temperature ramp stage of the TPD experiment. This particular point in our consistent methodology provides the connection between the uptake and desorption stage of the TPD experiment.

The complete history of the TPD experiment, including adsorption, desorption, and any intermediate annealing periods, can be followed with the consistent methodology above. There are two primary principles in this approach: (i) A consistent treatment of all the experimental stages of the TPD experiment, so that all the processes are always accounted for. Only the relative importance of the processes changes between the experimental stages. (ii) The final conditions (concentration profile) calculated at the end of each experimental stage are used as the initial conditions (concentration profile) for the simulation of the stage following immediately in the experimental protocol. Therefore, both the adsorption and permeation (isothermal exposure) and the desorption (TPD) experiments are modeled in a uniform and consistent way. Previous work had focused on modeling either permeation or $\mathrm{TPD}^{17}$ alone. To the best of our knowledge no one has attempted to link the analysis and interpretation of both experimental stages. The major advantage of this new approach is the elimination of any arbitrary decisions or inaccurate approximations regarding the concentration profiles achieved at the end of the isothermal exposure stage. The finite-medium formulation of the problem, introduced with the present model, is an additional advantage of this new methodology.

Our model involves the simultaneous solution of coupled partial and ordinary differential equations (PDE and ODEs). Analytical closed-form solutions to the equations of the model above are not feasible, and therefore the model has to be analyzed numerically. A second-order accurate finitedifferences scheme with automatically adjustable time step has been designed and implemented for the solution of the equations (for details, see Refs. 18-20).
TABLE I. Parameter values for the model, giving the best agreement between calculated and experimental (Ref. 21) TPD spectra for the $\mathrm{H}_{2} / \mathrm{Pd}(110)$ system. Values for the parameters: $E_{d}^{*}, F_{d}, E_{D}^{*}, D_{0}, X_{3 s}$, and $S_{0}$ were taken from the literature (Ref. 24). Results of the calculations are shown in Figs. 2, 3, 4, and 6.

\begin{tabular}{ll}
\hline \hline$E_{d}^{*}=24 \mathrm{kcal} / \mathrm{mol}$ & $F_{d}=8 \times 10^{-2} \mathrm{~cm}^{2} /$ atom $/ \mathrm{s}$ \\
$E_{21}^{*}=5 \mathrm{kcal} / \mathrm{mol}$ & $F_{21}=1.5 \times 10^{4} \mathrm{~s}^{-1}$ \\
$E_{12}^{*}=8 \mathrm{kcal} / \mathrm{mol}$ & $F_{12}=1.5 \times 10^{4} \mathrm{~s}^{-1}$ \\
$E_{32}^{*}=5 \mathrm{kcal} / \mathrm{mol}$ & $F_{32}=1.5 \times 10^{4} \mathrm{~s}^{-1}$ \\
$E_{23}^{*}=8 \mathrm{kcal} / \mathrm{mol}$ & $F_{23}=1.5 \times 10^{4} \mathrm{~s}^{-1}$ \\
$E_{D}^{*}=5 \mathrm{kcal} / \mathrm{mol}$ & $D_{0}=2.83 \times 10^{-3} \mathrm{~cm}^{2} / \mathrm{s}$ \\
$Y_{s}=9.4 \times 10^{16} \mathrm{sites} / \mathrm{cm}^{3}$ & $X_{2 s}=4.7 \times 10^{14} \mathrm{sites} / \mathrm{cm}^{2}$ \\
$X_{3 s}=9.4 \times 10^{14}$ sites $/ \mathrm{cm}^{2}$ & $S_{0}=0.7$ \\
$L=0.1 \mathrm{~cm}$ & $\beta=2.83 \mathrm{~K} / \mathrm{s}$ \\
$m=3.32 \times 10^{-24}$ g/atom & $P($ desorption $)=1 \times 10^{-10}$ Torr \\
\hline \hline
\end{tabular}

\section{RESULTS AND DISCUSSION}

The primary goal of this work is to elucidate a onedimensional potential energy diagram, a set of frequency factors, and site densities of the three reservoirs involved, that would yield calculated TPD spectra as close as possible to the available experimental data. ${ }^{21}$ A secondary goal is the demonstration of several important new concepts which are essential for the correct interpretation of TPD spectra in RD systems, and emphasize the distinction between typical Langmuirian and RD systems.

All the results presented here were derived using values for the model's parameters as given in Table I. Values for the parameters: $E_{d}^{*}, F_{d}, E_{D}^{*}, D_{0}, X_{3 s}$, and $S_{0}$ were compiled from the literature. ${ }^{24}$ The specific value of the heating rate $(\beta)$ was selected so that the nonexponential part of the frequency factor for the diffusion in the bulk, $D_{0}$, is canceled out. The rest of the parameter values in Table I represent the set of values that gave simulated TPD spectra closest to the experimental spectra. Two comments are appropriate here: (1) The site density of the bulk $\left(Y_{s}\right)$ is such that for the normal density of pure $\mathrm{Pd},{ }^{24,25}$ it would provide one absorption site per $10^{7}$ palladium atoms. Therefore, our calculations are performed at the dilute limit of the hydrogen-palladium system, even when the normalized concentration is approaching the saturation limit of the bulk $(Y=1)$. Under such conditions, it is physically correct to use a frequency factor $\left(D_{0}\right)$ independent of concentration. (2) The frequency factors of the four rate processes connecting subsurface with surface and bulk are all of the order of magnitude of $10^{4} \mathrm{~s}^{-1}$, which is relatively low with respect to the typical value of $10^{13} \mathrm{~s}^{-1}$. Despite persistent efforts, we were unable to derive TPD spectra similar to the experimental results by using preexponential factors greater than $10^{13} \mathrm{~s}^{-1}$ or between $10^{4} \mathrm{~s}^{-1}$ and $10^{13} \mathrm{~s}^{-1}$. Similar low values of frequency factors have been reported elsewhere, ${ }^{26}$ and are generally observed for processes with substantially restricted transition states which have characteristically small activation entropies. ${ }^{27}$ This result strongly suggests that jumping between subsurface and surface or subsurface and bulk is a process characterized by a restricted transition state. Abnormally low frequency factors have been reported for nonadiabatic reactions; for ex- 


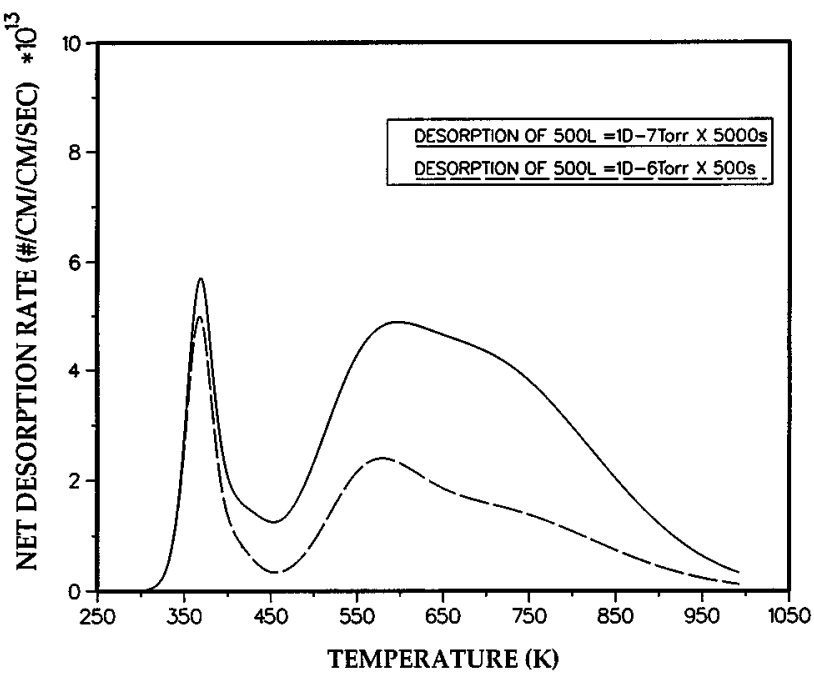

FIG. 2. Asymmetry of exposure time and pressure as demonstrated by the different TPD spectra following two nominally identical but historically different exposures. Adsorption temperature is $293 \mathrm{~K}$.

ample, the cis-trans isomerization of ethylenic derivatives. ${ }^{26}$ Recent work has clearly shown the strong effects of nonadiabaticity on the hydrogen diffusion in metals, and specifically in palladium. ${ }^{27}$ Therefore, low pre-exponential factors could be attributed to the strong nonadiabatic character of the processes connecting subsurface with surface and bulk. It is interesting to notice that pre-exponential factors as low as $10^{6} \mathrm{~s}^{-1}$ have also been reported for muons in $\mathrm{Cu}^{28}$ Similar theoretical speculations for low frequency factors characterizing proton transfer processes strongly related with tunneling effects have also appeared in the literature ${ }^{29}$ Overall, a frequency factor as low as $10^{4} \mathrm{~s}^{-1}$ is consistent with the current state of theory regarding diffusion of hydrogen in metals.

All the isothermal exposures were simulated for room temperature $(293 \mathrm{~K})$, so that we can directly compare our results with the available experimental data. Moreover, it is well known that hydrogen diffuses readily into the bulk of the $\operatorname{Pd}(110)$ single crystal at this temperature, offering the opportunity to study the effect of coupling diffusion to surface kinetics.

First, the importance of modeling the exact history of a TPD experiment is demonstrated. More specifically, Fig. 2 shows the desorption spectra as calculated from our model, for two different exposure schedules. One spectrum corresponds to the desorption of $500 \mathrm{~L}$ exposure achieved by exposing the crystal to $10^{-7}$ Torr for $5000 \mathrm{~s}$ (long-time exposure). The other spectrum corresponds to the desorption of $500 \mathrm{~L}$ exposure achieved by exposing the crystal to $10^{-6}$ Torr for $500 \mathrm{~s}$ (short-time exposure). Even though the nominal value of the two exposures is the same $(500 \mathrm{~L})$, the resulting TPD spectra show substantial differences. Recall that for typical Langmuir systems exposure pressure and time play a symmetric role, and any possible combination of exposure pressure and time resulting in the same nominal exposure gives identical desorption spectra. However, for

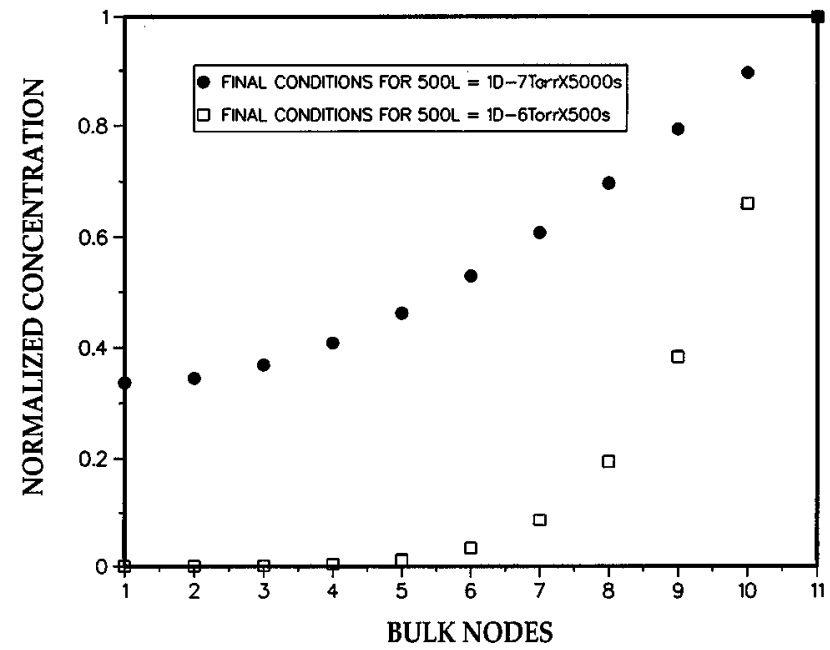

FIG. 3. Normalized bulk concentration profiles at the end of the two exposures used for the results of Fig. 2. Node 1 corresponds to the isolated end of the crystal $(Z=0)$, while node 11 corresponds to the bulk-subsurface interface.

RD systems the symmetry between exposure pressure and time is broken. A direct consequence of this fact is that for the correct interpretation of TPD spectra of RD systems, one needs to take into account not only the nominal exposure value but also the particular exposure pressure and exposure time used. ${ }^{18,20}$

The difference between the two spectra shown in Fig. 2 reflects the substantial difference in the amount of hydrogen absorbed into the subsurface and bulk of the crystal during the two distinct exposure schedules. The areas under the two desorption spectra suggest that the long-time $500 \mathrm{~L}$ exposure has resulted in much more hydrogen absorbed in the crystal, in comparison with the corresponding amount at the end of the short-time $500 \mathrm{~L}$ exposure. The first desorption peak of the two spectra, attributed to the desorption of surface chemisorbed hydrogen, does not change significantly between the two exposure schedules. Therefore, the amount of hydrogen diffusing in the subsurface and bulk of the crystal depends strongly on the exposure schedule. In support of this argument, Fig. 3 shows the normalized concentration profiles of the bulk at the end of the isothermal exposure schedules resulting in the TPD spectra of Fig. 2. The short-time $500 \mathrm{~L}$ exposure leaves the bottom-half of the crystal almost void of hydrogen. On the other hand, the long-time $500 \mathrm{~L}$ exposure allows more time $(5000 \mathrm{~s})$ for hydrogen to diffuse into the whole crystal, even though the gas phase hydrogen pressure is one order of magnitude less than its value in the short-time exposure. Figure 3 shows that the top layers of the bulk approached saturation, and the bottom layers reached more than $30 \%$ of their saturation capacity by the end of the longtime exposure. Furthermore, calculation of almost identical TPD spectra of the $\mathrm{H}_{2} / \mathrm{Pd}(110)$ system for two exposure schedules with identical exposure time but exposure pressures different by an order of magnitude, proves that exposure time, and not exposure pressure, is the dominant variable (Fig. 4). 


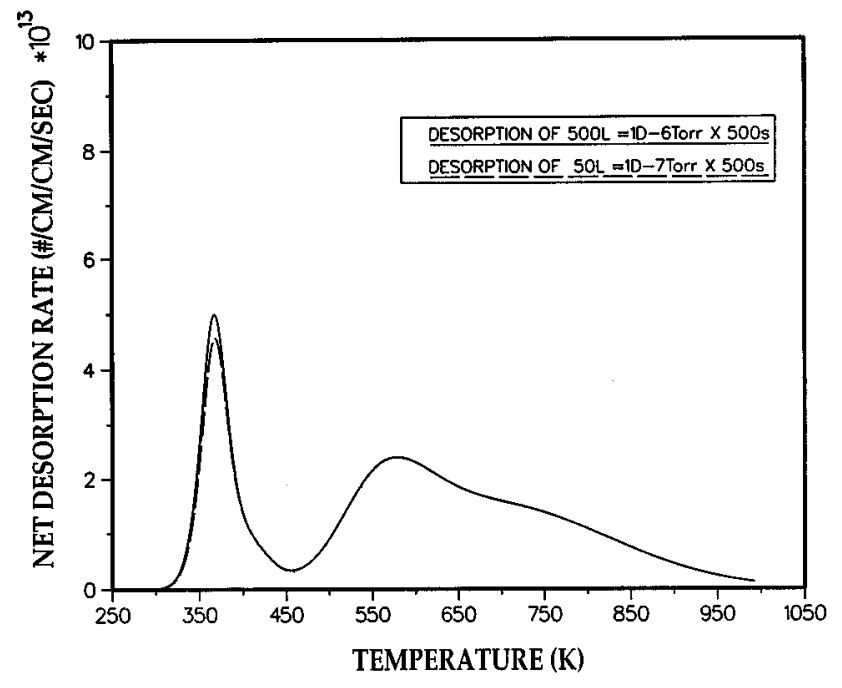

FIG. 4. Desorption spectra following two different exposures. Adsorption temperature is $293 \mathrm{~K}$. Exposure time is identical, but exposure pressure is changing by one order of magnitude. The effect of exposure pressure is not substantial.

Comparison between the experimental data ${ }^{21}$ and the corresponding results of our model (Fig. 5) indicates a good agreement, at least qualitatively. The first peak is due to the desorption of the surface chemisorbed hydrogen, while the second broad peak represents the desorption of hydrogen that initially diffused into the subsurface and bulk of the crystal. The original experimental work ${ }^{21}$ does not report on the exact exposure pressure and time used. Therefore, for the sake of comparison, we arbitrarily chose a hydrogen exposure pressure of $10^{-7}$ Torr to perform our calculations. Since there is an infinite number of possible pressure-time combinations that would yield any specific nominal exposure, it is conceivable that our choice of values might not be the best for obtaining the closest agreement between calculated and experimental data. Moreover, possible annealing periods be-

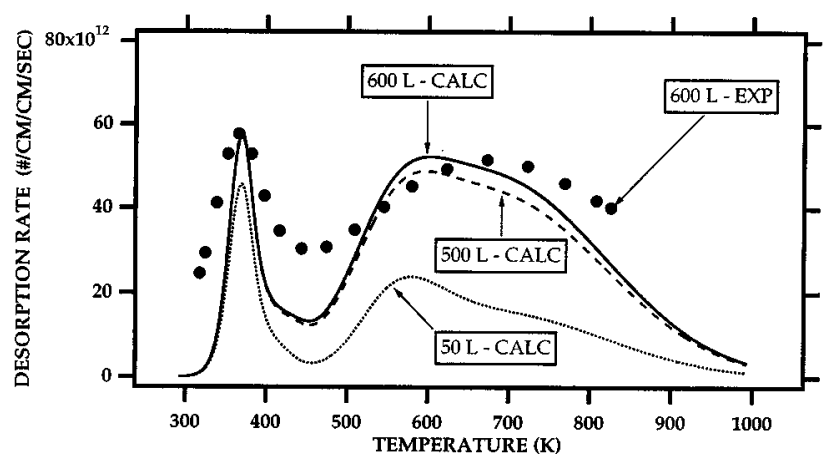

FIG. 5. Modeling results for the $\mathrm{H}_{2} / \mathrm{Pd}(110)$ system. Exposure set at $293 \mathrm{~K}$, with model parameter values as given in Table I and exposure pressure of $10^{-7}$ Torr. Experimental TPD data (reproduced with permission from Ref. 21) are also shown as discrete points $(600 \mathrm{~L})$. The original experimental data have been scaled so that the maxima of the low temperature peak of the experimental and calculated $600 \mathrm{~L}$ spectra coincide with each other. tween the exposure and temperature ramp can be of particular importance, since the adsorbates and absorbed species would have the time to redistribute into and on the crystal. Nevertheless, comparison between the experimental and the calculated desorption spectra for the $600 \mathrm{~L}$ exposure (marked points and continuous line in Fig. 5, respectively) indicates that our calculations capture the main features of the experimental results.

Previous experimental work beyond $\mathrm{TPD}^{31}$ has suggested evidence of a distinct subsurface hydrogen state in palladium with electronic and vibrational properties different from the properties of the surface and bulk states. A distinct subsurface state has also been suggested by recent theoretical work for the $\mathrm{H}_{2} / \mathrm{Pd}(100),{ }^{32} \quad \mathrm{H}_{2} / \mathrm{Pd}(111),{ }^{27(b), 30}$ and $\mathrm{H}_{2} / \mathrm{Pd}(110)^{33}$ systems. These $a b$ initio type of calculations for the $\operatorname{Pd}(100)$ and $\operatorname{Pd}(110)$ surfaces focused on the relative stability ("well-depths") of the different adsorption sites, with no particular emphasis on the related transition states (surface/subsurface and subsurface/bulk). Salahub and co-workers $^{33}$ focused on resolving the $(2 \times 1) \mathrm{H}$-covered $\operatorname{Pd}(110)$ surface structure, while Tomanek et al. ${ }^{33}$ analyzed the same surface but for coverages less than 1, where the effect of the subsurface state is not expected to be very important. Hence, it is only with results for the $\mathrm{Pd}(111)$ surface that we can compare our activation energy barriers. The fact that the original experimental work ${ }^{21}$ refers to the observed similarities between the broad peak position for $\operatorname{Pd}(110)$ and $\operatorname{Pd}(111)$ surfaces for the $600 \mathrm{~L}$ exposure, makes this comparison more meaningful. Paul and Sautet ${ }^{30}$ found that the barrier for the surface-subsurface transition is about 7.6 $\mathrm{kcal} / \mathrm{mol}(=0.33 \mathrm{eV})$, which is in good agreement with our results $\left(E_{32}^{*}=5 \mathrm{kcal} / \mathrm{mol}\right.$ and $\left.E_{23}^{*}=8 \mathrm{kcal} / \mathrm{mol}\right)$, given the fact that these values refer to different surfaces. Doll and co-workers ${ }^{27(b)}$ suggest that the rigid-lattice or nonadiabatic barrier for the same transition in $\operatorname{Pd}(111)$ is $4.2 \mathrm{kcal} / \mathrm{mol}$ $(=0.181 \mathrm{eV})$. This particular nonadiabatic character of the surface-subsurface transition has been suggested based on the unusually low pre-exponents mentioned above [calculated in this work, and in Ref. 27(b)]. Considering the accuracy of both $a b$ initio work and our work, we conclude that the results presented here are in reasonable agreement with the $a b$ initio results available for the $\mathrm{H}_{2} / \mathrm{Pd}(111)$ system.

\section{SUMMARY AND CONCLUSIONS}

The consistent approach introduced here involves modeling both isothermal adsorption and dissolution as well as temperature programmed desorption by using mean-field methods. Thus, the entire TPD experiment can be accurately simulated. Direct comparison between the experimental and modeling results has improved the current characterization of activation energies and frequency factors controlling the rate processes involved. Modeling experimental TPD spectra in conjunction with information provided by more microscopic methods such as spectroscopy and scattering experiments can improve the understanding of the $\mathrm{H}_{2} / \mathrm{Pd}$ system.

In particular, we have shown that for the $\mathrm{H}_{2} / \mathrm{Pd}$ system where surface processes are coupled to diffusion into the 
bulk, exposure time plays a determining role because diffusion is strongly time dependent. As a result, reporting both exposure pressure and exposure time are necessary for a complete understanding of coupled RD processes. An immediate consequence is that knowledge of the exact history of the experiment is necessary for the complete interpretation of the desorption spectra. Following the experimental history with our detailed model allowed the derivation of a simplified one-dimensional potential energy diagram, a set of frequency factors and site densities of the three reservoirs involved, that all together lead to calculated spectra in good agreement with the available experimental data.

The proposed one-dimensional potential energy diagram must include three distinct energetic states: surface, subsurface, and bulk. The subsurface state has been suggested by previous workers for the $\mathrm{H}_{2} / \mathrm{Pd}(110)$ system, but never tested within the framework of modeling TPD results. We concluded that the incorporation of a distinct subsurface state is required to obtain reasonable agreement between the model and experimental TPD data. The restricted nature of the surface-subsurface and subsurface-bulk transition states is emphatically demonstrated by the unusually low frequency factors calculated for the rate processes connecting subsurface with bulk and subsurface with surface. Further agreement between the activation energy barriers for the surfacesubsurface transition calculated here and the results of other theoretical work for the $\mathrm{H}_{2} / \mathrm{Pd}(111)$ system $^{27(b), 30}$ supports the value of mean-field modeling for such complicated systems.

The good agreement between the results of our calculations and the experimental TPD data for the $\operatorname{Pd}(110)$ surface strongly encourages the application of the methodology developed to the interpretation of TPD spectra of hydrogen from other palladium surfaces. The approach presented here holds a strong promise for understanding the effect of bulk hydrogen in palladium catalysis. In addition, catalytic mechanisms with other metals which involve the diffusion of adsorbed species into the subsurface and bulk of the catalysts can be characterized in considerable detail by combining modeling with experiments.

\section{ACKNOWLEDGMENTS}

Financial support from SRC, ARPA, IBM, and a Rackham Predoctoral Fellowship (M.M.) is gratefully appreciated.

${ }^{1} \mathrm{P}$. Gray and S. K. Scott, Chemical Oscillations and Instabilities (Clarendon, Oxford, 1990).

${ }^{2}$ G. Nicolis and I. Prigogine, Self-Organization in Nonequilibrium Systems (Wiley, New York, 1977).

${ }^{3}$ R. J. Field and M. Burger, eds., Oscillations and Traveling Waves in Chemical Systems (Wiley-Interscience, New York, 1985).

${ }^{4}$ P. Gray, G. Nicolis, F. Baras, P. Borckmans, and S. K. Scott, eds., Spatial Inhomogeneities and Transient Behaviour in Chemical Kinetics (Manchester University, Manchester and New York, 1990).
${ }^{5}$ J. D. Murray, Mathematical Biology (Springer-Verlag, Berlin and Heidelberg, 1989).

${ }^{6}$ J. D. Murray, Sci. Amer. 258(3), 80 (1988).

${ }^{7}$ P. C. Fife, Dynamics of Internal Layers and Diffusive Interfaces (SIAM, Philadelphia, PA, 1988).

${ }^{8}$ J. P. Keener, Waves in Excitable Media, SIAM J. Appl. Math. 39, 528 (1980).

${ }^{9}$ J. Smoller, Shock Waves and Reaction-Diffusion Equations (SpringerVerlag, Berlin, Heidelberg, New York, and Tokyo, 1983).

${ }^{10} \mathrm{R}$. Aris, The Mathematical Theory of Diffusion and Reaction in Permeable Catalysts, Vols. I and II (Clarendon, Oxford, 1975).

${ }^{11}$ H. C. Tuckwell, Science 205, 493 (1979).

${ }^{12}$ W. H. Press, B. P. Flannery, S. A. Teukolsky, and W. T. Vetterling, Numerical Recipes (Cambridge University Press, Cambridge, MA, 1989).

${ }^{13}$ G. D. Smith, Numerical Solution of Partial Differential Equations (Clarendon, Oxford, 1985).

${ }^{14} \mathrm{~J}$. Stoer and R. Bulirsch, Introduction to Numerical Analysis (SpringerVerlag, New York, 1980)

${ }^{15}$ P. A. Redhead, Vacuum, 12, 203 (1962); R. J. Cvetanovic and Y. Amenomiya, Advan. Catal. 17, 103 (1967).

${ }^{16}$ A. M. de Jong and J. W. Niemantverdriet, Surf. Sci. 233, 355 (1990).

${ }^{17}$ K. J. Leary, J. N. Michaels, and A. M. Stacy, AIChE J. 34, 263 (1988); B. D. Kay, C. H. F. Peden, and D. W. Goodman, Phys. Rev. B 34, 817 (1986); J. W. Davenport, G. J. Dienes, and R. A. Johnson, ibid. 25, 2165 (1982); M. A. Pick, ibid. 24, 4287 (1981); M. A. Pick, J. W. Davenport, M. Strongin, and G. J. Dienes, Phys. Rev. Lett. 43, 286 (1979); V. P. Zhdanov, Surf. Sci. 179, L57 (1987); V. P. Zhdanov and P. R. Norton, Langmuir 10, 1292 (1994); A. L. Cabrera, J. Chem. Phys. 93, 2854 (1990); A. L. Cabrera, E. Morales, and J. N. Armor, J. Mater. Res. 10, 779 (1995).

${ }^{18}$ M. Mavrikakis, J. W. Schwank, and J. L. Gland, Surf. Sci. 355, L385 (1996).

${ }^{19}$ M. Mavrikakis, J. W. Schwank, and J. L. Gland, J. Phys. Chem. 100, 11389 (1996)

${ }^{20}$ M. Mavrikakis, Ph.D. thesis, University of Michigan, 1994.

${ }^{21}$ H. Conrad, G. Ertl, and E. E. Latta, Surf. Sci. 41, 435 (1974).

${ }^{22}$ B. S. Kang and K. S. Sohn, Physica B 205, 163 (1995); S. W. Rick, D. L. Lynch, and J. D. Doll, J. Chem. Phys. 99, 8183 (1993); M. Lagos, Surf. Sci. 122, L601 (1982); M. Lagos and I. K. Schuller, ibid. 138, L161 (1984).

${ }^{23}$ M. G. Cattania, V. Penka, R. J. Behm, K. Christmann, and G. Ertl, Surf. Sci. 126, 382 (1983).

${ }^{24}$ (a) Z. Paal and P. G. Menon, eds., Hydrogen Effects in Catalysis (Marcel Dekker, New York, 1988), and references therein; (b) G. Alefeld and J. Volkl, eds., Hydrogen in Metals I \& II (Springer-Verlag, New York, 1978), and references therein.

${ }^{25}$ F. A. Lewis, The Palladium Hydrogen System (Academic, London, 1967).

${ }^{26} \mathrm{~S}$. Glasstone, K. J. Laidler, and H. Eyring, The Theory of Rate Processes (McGraw-Hill, New York, 1941).

${ }^{27}$ (a) Y. Li and G. Wahnstrom, Phys. Rev. B 46, 14528 (1992); (b) S. W. Rick, D. L. Lynch, and J. D. Doll, J. Chem. Phys. 99, 8183 (1993).

${ }^{28}$ I. I. Gurevich, E. A. Meleshko, I. A. Muratova, B. A. Nikolsky, V. S. Roganov, V. I. Selivanov, and B. V. Sokolov, Phys. Lett. 40A, 143 (1972).

${ }^{29}$ K. J. Laidler, Theories of Chemical Reaction Rates (McGraw-Hill, New York, 1969).

${ }^{30}$ J. F. Paul and P. Sautet, Phys. Rev. B 53, 8015 (1996).

${ }^{31}$ U. Stuhr, H. Wipf, T. J. Udovic, J. Weissmuller, and H. Gleiter, J. Phys.: Condens. Matter 7, 219 (1995); J. M. Nicol, J. J. Rush, and R. D. Kelley, Phys. Rev. B 36, 9315 (1987).

${ }^{32}$ T. L. Einstein, M. S. Daw, and S. M. Foiles, Surf. Sci. 227, 114 (1990); D. Hennig, S. Wilke, R. Lober, and M. Methfessel, ibid. 287/288, 89 (1993); S. Wilke, D. Hennig, R. Lober, M. Methfessel, and M. Scheffler, ibid. 307-309, 76 (1994); S. Wilke, D. Hennig, and R. Lober, Phys. Rev. B 50, 2548 (1994)

${ }^{33}$ I. Papai, D. R. Salahub, and C. Mijoule, Surf. Sci. 236, 241 (1990); D. Tomanek, Z. Sun, and S. G. Louie, Phys. Rev. B 43, 4699 (1991). 